\title{
Serum metabolic effects of corn oligopeptides with 7-day supplementation on early post-surgery primary liver cancer patients: a double-blind randomized controlled trial
}

\author{
Weiqi Rong $^{1 \#}$, Hui Xia ${ }^{2 \#}$, Kai Zhang ${ }^{3 \#}$, Yihan Zhang ${ }^{2 \#}$, Changcheng Tao ${ }^{1}$, Fan Wu ${ }^{1}$, Liming Wang ${ }^{1}$, \\ Hong Zhang', Guiju Sun ${ }^{2}$, Jianxiong Wu ${ }^{1}$
}

${ }^{1}$ Department of Hepatobiliary Surgery, National Cancer Center/National Clinical Research Center for Cancer/Cancer Hospital, Chinese Academy of Medical Sciences (CAMS) and Peking Union Medical College (PUMC), Beijing, China; ${ }^{2}$ Key Laboratory of Environmental Medicine and Engineering of Ministry of Education, Department of Nutrition and Food Hygiene, School of Public Health, Southeast University, Nanjing, China; ${ }^{3}$ Department of Interventional Therapy, Tianjin Medical University Cancer Institute and Hospital, National Clinical Research Center for Cancer, Key Laboratory of Cancer Prevention and Therapy, Tianjin's Clinical Research Center for Cancer, Tianjin, China

Contributions: (I) Conception and design: J Wu, G Sun, W Rong, H Xia, Y Zhang; (II) Administrative support: J Wu, W Rong, K Zhang, C Tao; (III) Provision of study materials or patients: K Zhang, F Wu, L Wang; (IV) Collection and assembly of data: J Wu, W Rong, K Zhang; (V) Data analysis and interpretation: G Sun, H Xia, Y Zhang, H Zhang; (VI) Manuscript writing: All authors; (VII) Final approval of manuscript: All authors.

\#These authors contributed equally to this work.

Correspondence to: Jianxiong Wu. Department of Hepatobiliary Surgery, National Cancer Center/National Clinical Research Center for Cancer/ Cancer Hospital, Chinese Academy of Medical Sciences (CAMS) and Peking Union Medical College (PUMC), Beijing 100021, China. Email: dr_wujx@163.com; Guiju Sun. Key Laboratory of Environmental Medicine and Engineering of Ministry of Education, Department of Nutrition and Food Hygiene, School of Public Health, Southeast University, Nanjing 210009, China. Email: gjsun@seu.edu.cn.

Background: Liver cancer as the main leading cancer has caused heavy burdens globally. The prognosis of liver cancer is closely related with postoperative nutrition support. Corn oligopeptides (COPs) are protein hydrolysates produced by enzymatic treatments, which have shown potential bioactivities, such as inhibiting angiotensin I-converting enzyme, resisting lipid peroxidation and anti-oxidant. However, the correlation between COPs and liver cancer patients is still unknown and the potential mechanism of COPs on liver cancer is unclear as well. The aim of this study was to assess effects of 7-day intervention of COPs after surgery on liver function and serum metabolic profiles of liver cancer patients.

Methods: Patients were assigned into COPs intervention group $(n=50)$ and control group $(n=91)$ for 7 days. Investigations were scheduled at $1^{\text {st }}$ day and $7^{\text {th }}$ day after liver resection surgery respectively, mainly including anthropometric, biochemical indexes and liquid chromatography-mass spectrometry (LC/MS) analysis.

Results: Seven-day supplementation of COPs on early post-surgery liver cancer patients down-regulated levels of alanine aminotransferase, aspartate aminotransferase, total bilirubin, direct bilirubin and upregulated prothrombin time activity and prealbumin levels. LC/MS analysis revealed metabolic signatures including regulation of 16 metabolites, which was closely related with two metabolic pathways (nicotinate and nicotinamide metabolism, fatty acid metabolism).

Conclusions: COPs supplementation has displayed the potentials on alleviating the injury of liver function and it may be due to regulation of fatty acid metabolism, nicotinate and nicotinamide metabolism, lipid peroxidation and anti-inflammatory action. More researches are warranted in future to confirm the exact mechanisms.

Keywords: Liver cancer; corn oligopeptides supplementation; metabolomics; randomized controlled trial

Submitted Mar 16, 2021. Accepted for publication Aug 11, 2021.

doi: $10.21037 / \mathrm{hbsn}-21-116$

View this article at: https://dx.doi.org/10.21037/hbsn-21-116 


\section{Introduction}

Liver cancer is the sixth most commonly diagnosed cancer and the fourth leading cause of cancer death worldwide (1). 953,000 incident cases of liver cancer and 819,000 deaths were reported globally in 2017 (2). In China, liver cancer is the fourth most common malignancy and the third leading cause of tumor-related deaths (3). The mortality of liver cancer varies considerably across the world, with the highest observed in East Asia (4). Hepatectomy is the most common approach for achieving long-term survival for liver patients, which include hepatectomy and liver transplantation (3). Postoperative nutrition support often leads to better prognostic significance in clinical outcome in surgical patients diagnosed with cancer (5-7). Researchers have found that dietary natural products could be potential sources for the prevention and treatment of liver cancer (8).

Bioactive peptides have received great attention because of its beneficial effects on human health for many years (9). Researches in vitro and in vivo have demonstrated that bioactive peptides can exhibit anti-oxidant, angiotensinconverting enzyme-I inhibitory activity and antiinflammatory, anticancer and immunomodulatory (10-12). In addition, addition of protein-enriched enteral nutrition improved postoperative recovery for patients with primary liver cancer (7). Corn oligopeptides (COPs) are protein hydrolysates produced by enzymatic treatments (13). COPs have been reported to have significant bioactivities, such as inhibiting angiotensin I-converting enzyme (14), resisting lipid peroxidation (15), anti-oxidant (16) and facilitating alcohol metabolism (17). Animal experiments have shown that COPs alleviated hepatic tissue damage on alcoholic animal model and improved the abnormal lipid metabolism $(13,18)$. The in vitro study demonstrated that COPs significantly prevented the cytotoxicity and displayed potential hepatoprotection in ethanol-induced HepG2 cells (19).

Nutritional metabolomics was defined as "use of small molecule chemical profiling to integrate diet and nutrition in complex biosystems" (20). Nutritional metabolomics is now widely used for exploring the complicate connections between diet and the human organism (21). It provides nutrition assessment and potential relevant metabolic pathways by measuring small molecules, such as saliva, plasma, urine, tissues and blood (22). A study of whole grain carried out on 33 postmenopausal Finnish women demonstrated favorable changes in branched amino acid and single carbon metabolism (23). Another clinical trial measured 296 serum metabolites among healthy controls and T2D patients and identified the strongest biomarker of fish oil intake (24). The metabolomics approach could be used to explore the roles that COPs played in regulation metabolism of patient with liver cancer.

In summary, the additional nutritional support for liver cancer patients after surgery is necessary, especially for early post-surgery nutritional support. COPs as a functional peptide has displayed potentials on anti-cancers mostly reported in animal studies. However, there is no clinical study to the correlations between COPs and liver cancer so far. The present study aims to investigate the potential beneficial of COPs supplementation on liver cancer patients after surgery. We conducted supplementation of COPs among liver cancer patients after surgical resection to explore the regulation mechanisms of COPs during recovery through an untargeted metabolomics approach. We present the following article in accordance with the CONSORT reporting checklist (available at https://hbsn. amegroups.com/article/view/10.21037/hbsn-21-116/rc).

\section{Methods}

\section{Participants}

The trial was conducted in accordance with the Declaration of Helsinki (as revised in 2013). The study was approved by the Ethics Committee of National Cancer Center/Cancer Hospital, Chinese Academy of Medical Sciences and Peking Union Medical College (permission code: 17-035/1290) and the written informed consent was obtained from all patients. According to inclusion and exclusion criteria, 160 patients were included in the study. Patients were allocated according to a randomly generated number sequence to the control group (Group A) or to the intervention group (Group B). Neither patients nor surgeon, nor researcher knew whether the patient will be in the control or intervention group. Ultimately, 141 patients recruited from the Cancer Hospital Chinese Academy of Medical Sciences between November 2017 and July 2019. The Inclusion and the exclusion criteria were as follows.

The inclusion criteria: (I) aged 18-75 years old; (II) patients with primary liver cancer diagnosed by "Practice guidelines for the pathological diagnosis of primary liver cancer" [2015] (25), histopathology or cytology; (III) judged to be resectable (R0 resection) before the surgery; (IV) no evidence of distant metastasis; (V) prior systemic 
therapy was not accepted; (VI) liver function evaluated as Child-Pugh Class A and Child-Pugh status should be calculated based on clinical findings and laboratory results during the screening period; (VII) Eastern Cooperative Oncology Group (ECOG) performance status of 0 or 1; (VIII) Nutritional Risk Screening (NRS) $\leq 3$; (IX) no serious heart, lung, liver and kidney dysfunction, jaundice, ascites and digestive tract obstruction and no acute infection; (X) no chemotherapy and radiotherapy; (XI) adequate bone marrow, liver, and renal function as assessed by the following laboratory tests conducted within 7 days before randomization; (XII) signed informed consent; (XIII) life expectancy of at least 6 months.

The exclusion criteria: (I) a known history of immunodeficiency or autoimmune disease (e.g., rheumatoid arthritis, systemic lupus erythematosus, multiple sclerosis, insulin-dependent diabetes mellitus, etc.); (II) unstable angina or myocardial infarction within the past 6 months before randomization, or congestive heart failure with New York Heart Association (NYHA) class $\geq 2$; (III) uncontrolled hypertension (systolic blood pressure $>150 \mathrm{mmHg}$ or diastolic pressure $>90 \mathrm{mmHg}$ despite optimal medical management); (IV) abnormal coagulation function [international normalized ratio (INR) $>1.5$ or active partial thromboplastin time (APTT) $>1.5 \times$ upper limit of normal (ULN)], bleeding tendency, receiving thrombolytic, anticoagulant therapy or with known inherited or acquired bleeding and thrombosis, such as hemophilia, blood coagulation dysfunction, thrombocytopenia, the splenic function, etc.; (V) arterial or venous thrombotic or embolic events such as cerebrovascular accident (including transient ischemic attacks), deep vein thrombosis, or pulmonary embolism within 6 months before the start of study medication; (VI) with any history of other cancer in the past 3 years (except adequately treated basal cell or squamous cell skin carcinoma or in-situ cervix cancer); (VII) chemotherapy, radiotherapy, molecular targeted therapy, bio-immunotherapy, or hormone therapy within 1 month before randomization; (VIII) history of severe allergy; (IX) history of severe mental illness; (X) breast feeding or pregnancy; (XI) has participated in another clinical trial within 4 weeks; (XII) known history of human immunodeficiency virus infection or syphilis; (XIII) sepsis and other non-controlling infectious fever; (XIV) history of organ allograft and bone marrow transplant; (XV) history of substance abuse or alcohol abuse; (XVI) considered poor compliance or refused to sign the informed consent.

\section{Study design}

Figure 1 illustrates the study process; 19 patients were loss of follow-up, of which 1 patient did not meet inclusion criteria, due to personal reasons, 1 patient withdrew from the study, and 17 patients were absence of serum samples. Finally, all subjects were randomly assigned into corn oligopeptides (COPs) intervention group $(\mathrm{n}=50)$ and the control group $(\mathrm{n}=91)$. During the 7 -day intervention, all subjects were treated only with the same enteral nutritional powder and same parenteral nutrition, and no other food consumption was provided. Both groups received 20 kilocalories (kcal) $/ \mathrm{kg}$ daily, and enteral and parenteral nutrition provided $50 \%$ calories respectively. For enteral feeding, the intervention group (TI) was treated with Intacted Protein Enteral Nutrition Powder (Nutrison, Milupa GmbH, Germany) enriched with $8 \mathrm{~g}$ COPs (China National Research Institute of Food and Fermentation), and the control group (CI) received Intacted Protein Enteral Nutrition Powder along per day for 1 week after surgery. Investigations were scheduled at $1^{\text {st }}$ day and $7^{\text {th }}$ day after surgery. After a 12 -hour overnight fast, blood samples were collected in the peripheral vein for further analysis. Serum samples separated for the analysis of metabolites were frozen at $-80^{\circ} \mathrm{C}$.

\section{Detection of anthropometric and biochemical indexes}

A general health examination including registration of body weight, height, Smoke and drink was collected before the intervention. In addition, we examined the liver function [serum levels of aspartate transaminase (AST), alanine aminotransferase (ALT), total bilirubin (TBIL), prothrombin time (PT) and $\alpha$-fetoprotein (AFP)] as the main outcomes. Serum concentrations of total cholesterol (TC), triglyceride (TG), CA19-9 (a tumor marker), blood urea nitrogen (BUN), hemoglobin (HGB) were assessed as the secondary outcomes with an auto-analyzer.

\section{Extraction and derivatization of metabolites}

\section{Metabolites extraction}

$50 \mu \mathrm{L}$ of each serum sample was transferred to an EP tube. After the addition of $200 \mu \mathrm{L}$ of extract solution (acetonitrile: methanol $=1: 1$, containing isotopically-labelled internal standard mixture), the samples were vortexed for $30 \mathrm{~s}$, sonicated for $10 \mathrm{~min}$ in ice-water bath, and then incubated for $1 \mathrm{~h}$ at $-40^{\circ} \mathrm{C}$ to precipitate proteins. Then the sample was 


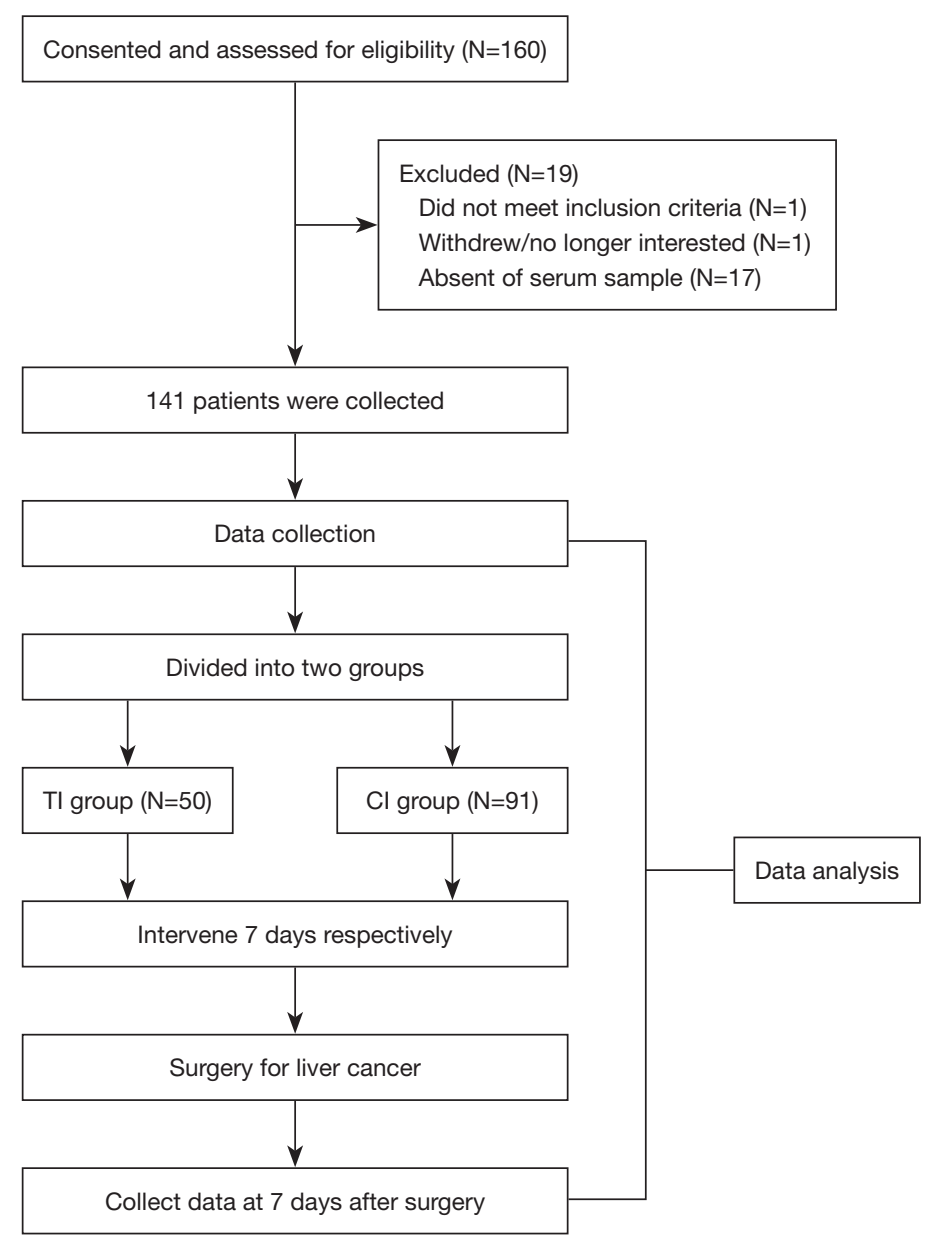

Figure 1 Participant flow throughout the study. TI, corn oligopeptides intervention group; CI, control group.

centrifuged at $12,000 \mathrm{rpm}$ for $15 \mathrm{~min}$ at $4{ }^{\circ} \mathrm{C}$. The resulting supernatant was transferred to a fresh glass vial for analysis. The quality control (QC) sample was prepared by mixing an equal aliquot of the supernatants from all samples.

\section{LC-MS/MS analysis}

LC-MS/MS analyses were performed using an UHPLC system (Vanquish, Thermo Fisher Scientific) with a UPLC BEH Amide column $(2.1 \mathrm{~mm} \times 100 \mathrm{~mm}, 1.7 \mu \mathrm{m})$ coupled to Q Exactive HFX mass spectrometer (Orbitrap MS, Thermo). The mobile phase consisted of $25 \mathrm{mmol} / \mathrm{L}$ ammonium acetate and 25 ammonia hydroxides in water $(\mathrm{pH}=9.75)$ (shown with A below) and acetonitrile (shown with $\mathrm{B}$ below). The analysis was carried with elution gradient as follows, $0-0.5 \mathrm{~min}$ with $95 \%$ of $\mathrm{B}, 0.5-7.0$ min with $95-65 \%$ of $\mathrm{B}, 7.0-8.0$ min with $65-40 \%$ of B, 8.0-9.0 min with $40 \%$ of B, 9.0-9.1 min with $40-95 \%$ of B and $9.1-12.0$ min with $95 \%$ of B. The column temperature was at $25^{\circ} \mathrm{C}$. The auto-sampler temperature was with $4^{\circ} \mathrm{C}$, and the injection volume was $3 \mu \mathrm{L}$.

The QE HFX mass spectrometer was equipped for its ability to acquire MS/MS spectra on informationdependent acquisition (IDA) mode in the control of the acquisition software (Xcalibur, Thermo). In this mode, the acquisition software continuously evaluates the full scan MS spectrum. The ESI source conditions were set as following, sheath gas flow rate as $50 \mathrm{Arb}$, Aux gas flow rate as $10 \mathrm{Arb}$, capillary temperature $320^{\circ} \mathrm{C}$, full MS resolution as 60,000 , MS/MS resolution as 7,500, collision energy as 10/30/60 in NCE mode, spray Voltage as $3.5 \mathrm{kV}$ (positive) or $-3.2 \mathrm{kV}$ (negative), respectively.

\section{Statistical analysis}

Social Sciences software Version 22.0 (SPSS) was used 
for the data analysis. All data are expressed as the mean \pm standard deviation. Continuous variables were compared using independent sample $t$-test or Wilcoxon tests, depending on the results of the normality test; categorical variables were compared using Chi-squared test, Fisher's exact test or Wilcoxon tests. The differential value of indicators between after intervention and baseline was used to compare the difference between TI and CI in order to reduce the effects of imbalance caused by baseline as well. A P value $<0.05$ was considered statistically significant. Multivariate statistical investigations containing principal component analysis (PCA) and orthogonal projections to latent structures-discriminant analysis (OPLS-DA) were carried out using SIMCA software (V15.0.2, Sartorius Stedim Data Analytics AB, Umea, Sweden). SIMCA software package was used to determine whether metabolites detected between groups show significant difference. The supervised OPLS-DA model was used to obtain maximal covariance between two groups. The changes of metabolites with variable importance and in projection (VIP) value $>1$ in the OPLS-DA model and the value $\mathrm{P}<0.05$ in the student's $t$-test were considered to be significantly different. Using Pearson's correlation analysis assess the correlations between serum indexes and metabolites, where $\mathrm{P}<0.05$ was considered significant.

\section{Results}

Basic information and effects of COPs on liver cancer patients

\section{Participants before intervention}

Of the 141 patients, the average age was $56.65 \pm 1.62$ years with the average body mass index (BMI) of 24.09 (22.32$26.49) \mathrm{kg} / \mathrm{m}^{2}$. Significant differences were observed between TI and CI in serum TBIL, direct bilirubin (DBIL) and prothrombin activity (PTA) levels $(\mathrm{P}<0.05)$. No difference was observed between TI and CI groups in volume of liver resection. No differences in anthropometric indexes and other serum indexes were observed [weight, BMI, ALT, AST, albumin (Alb), pre-Alb, creatinine (CREA), TC, TG, BUN, AFP, CA199, white blood cell (WBC), neutrophil (NE), red blood cell (RBC), HGB, platelet (PLT), lymphocyte (LY)], shown in Table 1. No differences were observed in smoke/drink information, ECOG performance status, histopathological scores (shown in Tables 2-4).

\section{Participants after surgery within $\mathbf{7}$ days}

Table 1 compares the serum indexes measured at 7 days after surgery. Serum ALT $(\mathrm{P}=0.01)$ and AST $(\mathrm{P}=0.01)$ levels show significant changes between TI and CI. No differences in other serum indexes. In addition, we compared the difference values of serum indexes between two investigations in $\mathrm{TI}$, to the difference values of serum indexes in $\mathrm{CI}$, serum $\operatorname{ALT}(\Delta \mathrm{P}=0.04)$, AST $(\Delta \mathrm{P}=0.03)$, TBIL $(\triangle \mathrm{P}=0.00)$, DBIL $(\Delta \mathrm{P}=0.01)$ levels decreased significantly, serum PTA $(\Delta \mathrm{P}=0.00)$, Pre-Alb $(\Delta \mathrm{P}=0.01)$ levels increased significantly. No adverse effects were observed.

\section{Metabolic profiling via liquid chromatography-mass spectrometry (LC/MS) analysis}

To assess systemic variations in small-molecule metabolites after intervention, we used an unbiased, non-targeted LC/ MS metabolomics approach. The total distribution of serum samples was shown in Figure 2 [red, blue, purple, yellow and green spots stand for treatment group before surgery in serum (TB), treatment group after surgery for 7 days in serum (TA), control group before surgery in serum (CB), control group after surgery for 7 days in serum (CA) and quality control (QC), respectively]. Figure 3 carried out the distribution with PCA and OPLSDA methods of each sample. We can see that at 7 days after surgery, the difference between TI and CI was larger than before surgery in serum $\{\mathrm{t}(1) \mathrm{p}[8.01 \%], \mathrm{t}(1)$ $\mathrm{p}[4.16 \%]\}$. Based on the quality set of VIP $>1$ and $\mathrm{P}<0.05$, 16 metabolites were identified. In the permutation test for OPLS-DA model of serum samples between TA and $\mathrm{CA}, \mathrm{R}^{2}$ and $\mathrm{Q}^{2}$ are very close to the slope of the $\mathrm{Y}$-axis, indicating that the model is robust (Figure 4). Among them the relative abundance of 1-Nitrohexane, 5,6-trans-25Hydroxyvitamin D3, Levetiracetam, Elaidic carnitine were decreased, relative abundance of 1-methylnicotinamide, L-palmitoylcarnitine, Panaquinquecol 1, LysoPE (16:0/0:0), Glycylproline, Monoglucuronylglycyrrhetinic acid, Heptadecanoyl carnitine, Stigmastane-3,6-dione, PC (20:4(8Z,11Z,14Z,17Z)/20:3(5Z,8Z,11Z)), alphatocopherolquinone, PC (22:6(4Z,7Z,10Z,13Z,16Z,19Z)/20: $1(11 Z)$ ), Naphthoherniarin were increased, shown in Table 5. The most significant pathways were plotted in terms of hypergeometric test $\mathrm{p}$ values (cube color) and impact (cube diameter) (Figure 5). Results showed two major pathways were altered upon COPS intervention: nicotinate and nicotinamide metabolism, fatty acid metabolism.

\section{Correlations between metabolites and serum indexes}

Figure 6 summarizes the association between metabolites 
Table 1 Anthropometric and biochemical effects of COPs on patients after surgery for 7 days

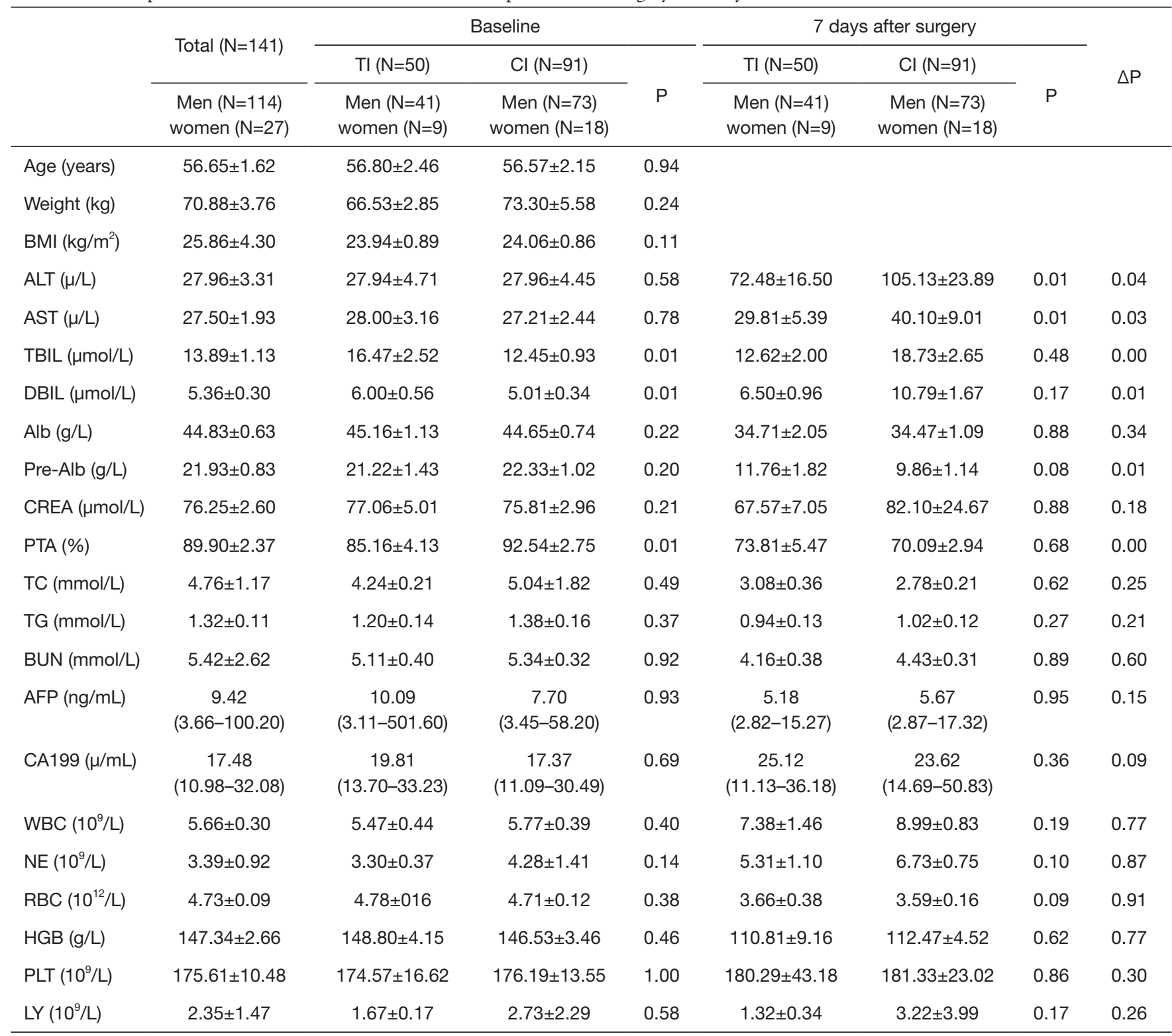

Data subjected to normal distribution are presented as the mean \pm standard deviation, otherwise expressed as median (inter quartile range). COPs, corn oligopeptides; TI, corn oligopeptides intervention group; $\mathrm{Cl}$, control group; $\mathrm{P}$, student $t$-test, considered as significance when $<0.05 ; \Delta \mathrm{P}$, the comparison between the difference of two investigations in $\mathrm{TI}$ and the difference of two measurements in Cl. BMI, body mass index; ALT, alanine aminotransferase; AST, aspartate aminotransferase; TBIL, total bilirubin; DBIL, direct bilirubin; Alb, albumin; Pre-Alb, Prealbumin; CREA, creatinine; PTA, prothrombin time activity; TC, serum total cholesterol; TG, triglyceride; BUN, blood urea nitrogen; AFP, alpha fetoprotein; CA199, carbohydrate antigen199; WBC, white blood cell; NE, neutrophil; RBC, red blood cell; HGB, hemoglobin; PLT, platelet; LY, lymphocyte.

and serum indexes. Spearman's correlation analysis showed that the relative abundance of 1-Methylnicotinamide was positively associated with the serum TG levels but negatively associated with the serum TBIL and DBIL levels. Relative abundance of L-palmitoylcarnitine was positively correlated with the serum LY levels and the relative abundance of 1-Nitrohexane was positively correlated with the serum NE levels. Relative abundance of LysoPE (16:0/0:0) was positively associated with the serum CA199 level, relative abundance of LY but negatively associated 
Table 2 Smoke/drink information in patients

\begin{tabular}{lccc}
\hline Group & Total $(\mathrm{N}=141)$ & $\mathrm{Tl}(\mathrm{N}=50)$ & $\mathrm{Cl}(\mathrm{N}=91)$ \\
\hline Smoke/drink & $78(55.30)$ & $26(52.00)$ & $52(57.10)$ \\
Non & $8(5.70)$ & $2(4.00)$ & $6(6.60)$ \\
Drink & $24(17.00)$ & $11(22.00)$ & $13(14.30)$ \\
Smoke & $31(22.00)$ & $11(22.00)$ & $20(22.00)$ \\
Both & & & 0.65 \\
\hline
\end{tabular}

$\mathrm{TI}$, Corn Oligopeptides intervention group; Cl, control group; P, student $t$-test, considered as significance when $<0.05$.

Table 3 ECOG performance status

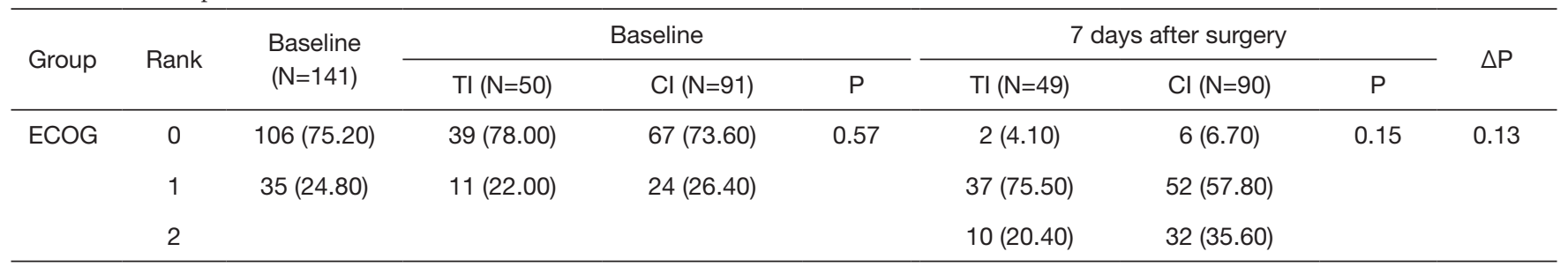

$\mathrm{Tl}$, Corn Oligopeptides intervention group; $\mathrm{Cl}$, control group; $\mathrm{P}$, student $t$-test, considered as significance when $<0.05$; $\Delta \mathrm{P}$, the comparison between the difference of two investigations in $\mathrm{TI}$ and the difference of two measurements in Cl. ECOG, Eastern Cooperative Oncology Group.

Table 4 The histopathological scores of liver cancer in patients

\begin{tabular}{|c|c|c|c|c|}
\hline Group & Rank & $\mathrm{TI}(\mathrm{N}=50)$ & $\mathrm{Cl}(\mathrm{N}=90)$ & $\mathrm{P}$ \\
\hline \multirow[t]{7}{*}{ Anatomy } & 1 & $6(12.00)$ & $10(11.10)$ & 0.47 \\
\hline & 2 & $18(36.00)$ & $34(37.80)$ & \\
\hline & 3 & $23(46.00)$ & $29(32.20)$ & \\
\hline & 4 & $2(4.00)$ & $3(3.30)$ & \\
\hline & 5 & $0(0.00)$ & $3(3.30)$ & \\
\hline & 6 & $1(2.00)$ & $8(8.90)$ & \\
\hline & 7 & $0(0.00)$ & $3(3.30)$ & \\
\hline \multirow[t]{7}{*}{ Microscope } & 1 & $6(12.00)$ & $12(13.30)$ & 0.93 \\
\hline & 2 & $15(30.00)$ & $31(34.40)$ & \\
\hline & 3 & $23(46.00)$ & $29(32.20)$ & \\
\hline & 4 & $4(8.00)$ & $5(5.60)$ & \\
\hline & 5 & $1(2.00)$ & $2(2.20)$ & \\
\hline & 6 & $1(2.00)$ & $9(10.00)$ & \\
\hline & 7 & $0(0.00)$ & $2(2.20)$ & \\
\hline
\end{tabular}

$\mathrm{TI}$, corn oligopeptides intervention group; $\mathrm{Cl}$, control group; $\mathrm{P}$, student $t$-test, considered as significance when $<0.05$. with the serum CREA, TBIL and DBIL levels. Relative abundance of Panaquinquecol 1 was positively related to the serum HGB level but negatively related to the serum BUN, CREA, PLT levels. Relative abundance of Glycylproline was positively associated with the serum CA199 level. Relative abundance of 5,6-trans-25-Hydroxyvitamin D3 was positively related to the serum AFP, BUN, DBIL, TBIL levels but negatively associated with the serum PTA level. Relative abundance of Levetiracetam was positively correlated with the serum BUN level but negatively correlated with the serum ALT and PTA levels. Additionally, relative abundance of Monoglucuronylglycyrrhetinic acid was positively related to the serum AFP, BUN, DBIL, NE, WBC levels but negatively related to the serum Alb, PTA and TG levels. Relative abundance of Stigmastane-3,6dione was positively associated with the serum HGB level but negatively associated with the serum CREA and PLT levels. Relative abundance of PC (20:4(8Z,11Z,14Z,17Z)/ 20:3(5Z,8Z,11Z)) was positively correlated with the serum CA199 and LY levels but negatively correlated with the serum ALT and TBIL levels. Relative abundance of alphatocopherolquinone was positively associated with the serum 


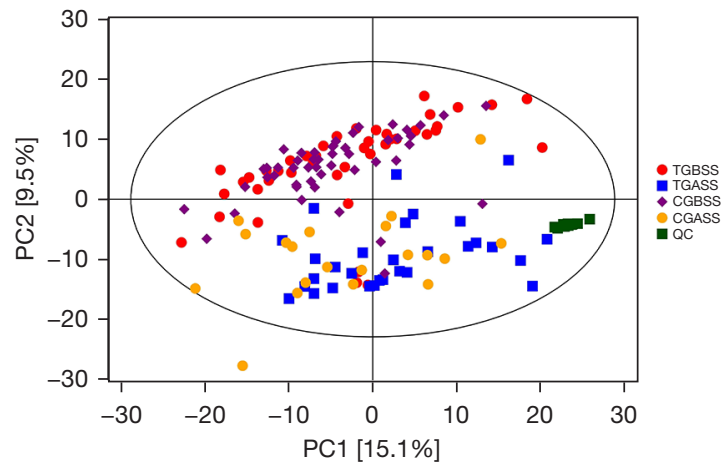

Figure 2 COPs intervention in patients affects the serum metabolome. The PCA score plots of total serum samples. TGBSS, treatment group before surgery in serum; CGBSS, control group before surgery in serum; TGASS, treatment group after surgery for 7 days in serum; CGASS, control group after surgery for 7 days in serum; QC, quality control; COPs, corn oligopeptides; PCA, principal component analysis.

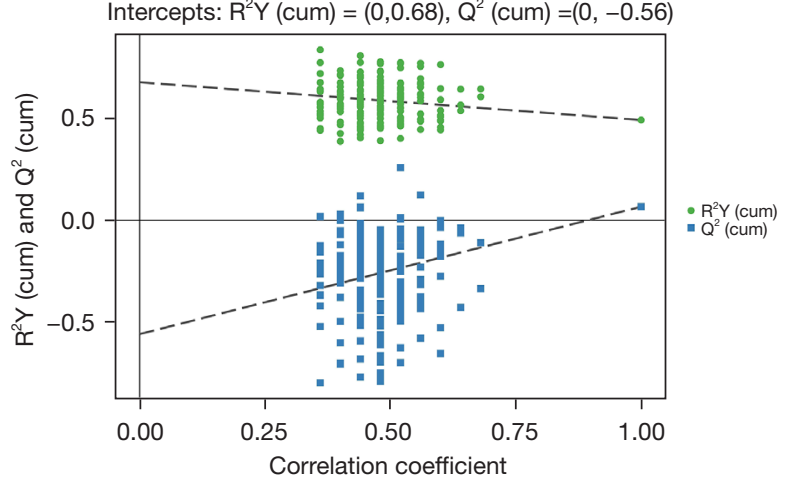

Figure 4 COPs intervention in patients affects the serum metabolome. Permutation test charts for OPLS-DA model of serum samples between TA and CA. TA, treatment group after surgery for 7 days in serum; CA, control group after surgery for 7 days in serum; COPs, corn oligopeptides; OPLS-DA, orthogonal projections to latent structures-discriminant analysis.
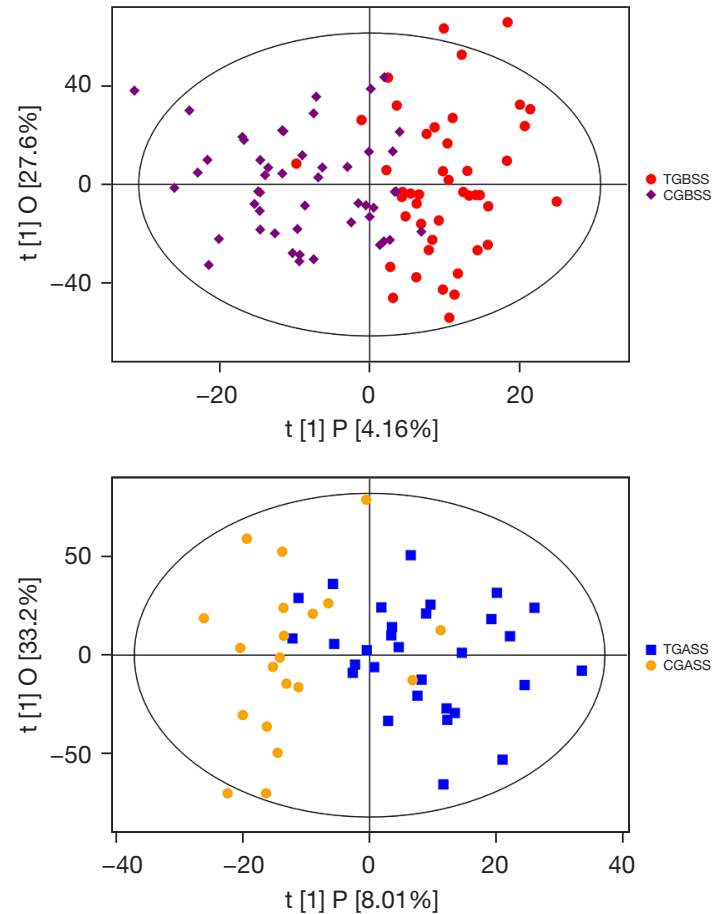

Figure 3 COPs intervention in patients affects the serum metabolome. The PCA (left) and OPLS-DA (right) score plots of serum. TGBSS, treatment group before surgery in serum; CGBSS, control group before surgery in serum; TGASS, treatment group after surgery for 7 days in serum; CGASS, control group after surgery for 7 days in serum; QC, quality control; COPs, corn oligopeptides; PCA, principal component analysis; OPLS-DA, orthogonal projections to latent structures-discriminant analysis. 
Table 5 Metabolite screen in serum between TA and CA

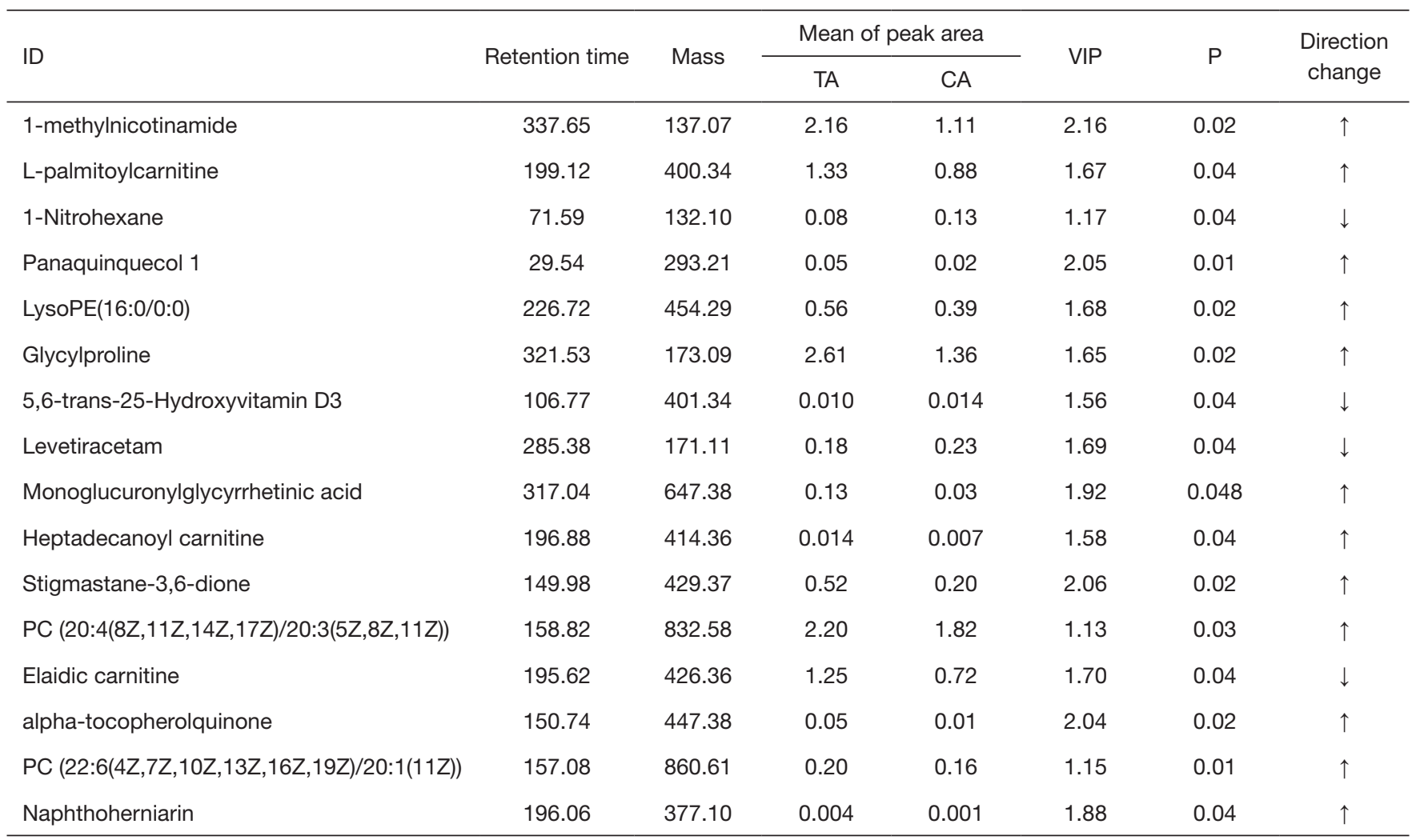

Mean peak of area, the average value of peak area normalization for each group; TA, treatment group after surgery for 7 days in serum; CA, control group after surgery for 7 days in serum; VIP, variable importance projection; P, student $t$-test, considered as significance when $<0.05$.

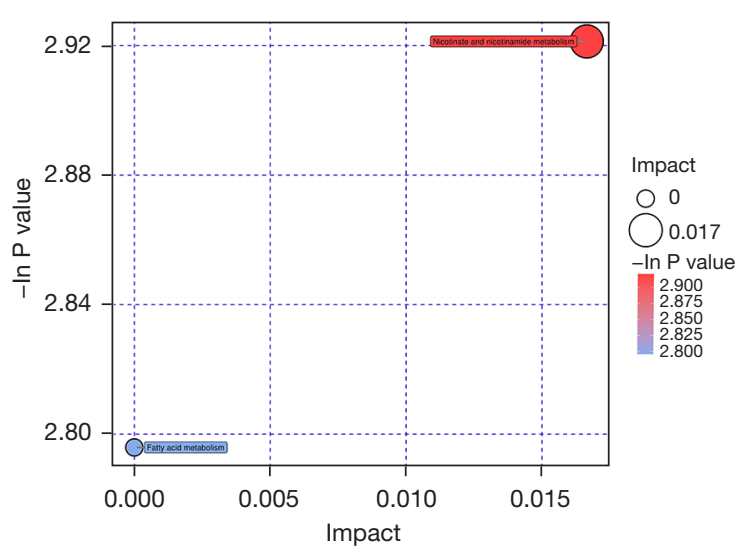

Figure 5 Pathway impact prediction of KEGG online database between TA and CA. The results of metabolic pathway analysis are presented in bubble diagram. The $\mathrm{P}$ value of enrichment analysis [negative natural logarithm, i.e., $-\ln (\mathrm{P})]$ positively correlated with the bubble color depth. TA, treatment group after surgery for 7 days in serum; CA, control group after surgery for 7 days in serum; KEGG, Kyoto Encyclopedia of Genes and Genomes.
HGB level but negatively correlated with the serum CREA and PLT levels. Relative abundance of PC (22:6(4Z,7Z,10Z, 13Z,16Z,19Z)/20:1(11Z)) was positively correlated with the serum CA199 and LY levels but negatively correlated with the serum ALT, TBIL and DBIL levels. Relative abundance of Naphthoherniarin was positively associated with the serum TBIL and DBIL levels but negatively associated with the serum BUN, CREA and PLT levels.

\section{Discussion}

In the present study, we applied LC/MS-based metabolic profiling to assess hepatoprotection of supplementation with COPs for 7 days in early post-surgery liver cancer patients. We found that COPs consumption could reduce the levels of ALT, AST, TBIL, DBIL and increase the levels of PTA and Pre-Alb. The LC/MS analysis revealed metabolic signatures by regulating sixteen metabolites and altering 


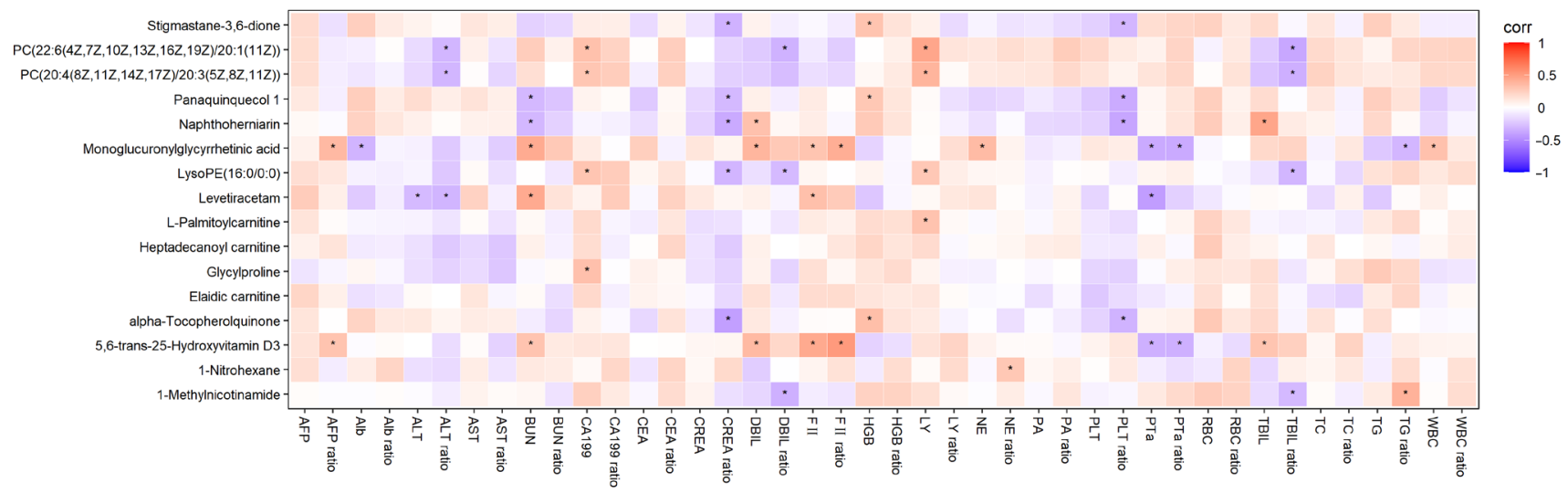

Figure 6 Correlation analysis between metabolites and serum indexes. Red (corr $=1)$, blue (corr $=-1)$, white $($ corr $=0)$ and, * represents a significant correlation $(\mathrm{P}<0.05)$. AFP, alpha fetoprotein; Alb, albumin; ALT, alanine aminotransferase; AST, aspartate aminotransferase; BUN, blood urea nitrogen; CA199, carbohydrate antigen 199; CEA, carcinoembryonic antigen; CREA, creatinine; DBIL, direct bilirubin; HGB, hemoglobin; LY, lymphocyte; NE, neutrophil; PA, prealbumin; PLT, platelet; PTa, prothrombin time activity; RBC, red blood cell; TBIL, total bilirubin; TC, serum total cholesterol; TG, triglyceride; WBC, white blood cell.

two metabolic pathways (nicotinate and nicotinamide metabolism, fatty acid metabolism).

ALT and AST are traditional liver markers, and the elevated serum levels indicated clear liver injury (26-28). In this study, serum AST and ALT levels increased between TI and CI after operation, but the augments of serum AST $(\mathrm{P}=0.01)$ and ALT $(\mathrm{P}=0.01)$ levels in TI were smaller than in $\mathrm{CI}$, which indicated lighter damage of liver function in TI. Furthermore, the serum levels of TBIL $(\triangle \mathrm{P}=0.00)$ were decreased in TI but increased in CI, serum DBIL $(\Delta \mathrm{P}=0.01)$ level elevated between two groups and showed similar tendency as the serum levels of ALT and AST. Liver injury markers TBIL and DBIL can sensitively reflect the extent of hepatocyte injury and liver parenchymal damage after surgery (29) and our results revealed that short-term COPs intervention may be beneficial for liver function recovering $(14,30)$. The levels of serum PTA and Pre-Alb reflect the injury of pathology of the liver and its synthetic function, thus an improvement of liver function indicated by an increasement in serum PTA and Pre-Alb levels $(31,32)$. Although serum PTA $(\Delta \mathrm{P}=0.00)$ and Pre-Alb $(\Delta \mathrm{P}=0.01)$ levels were decreased after operation in our study, the less reduction in TI group has revealed significantly hepatoprotection.

LC/MS metabolomics approach enabled the evaluation of the mechanisms of action of COPs. Nicotinamide $\mathrm{N}$-methyltransferase (NNMT), to produce 1-methylnicotinamide
(MNA), is predominantly expressed in the liver (33). NNMT has been reported to play a hepatoprotective compatible with the PGI (2)-releasing properties of MNA (34). MNA decreased liver cholesterol and TG levels, while the expression of lipogenic and cholesterol synthesis genes and the synthesis of fatty acid and cholesterol suppressed and regulates glucose, lipid and cholesterol metabolism by stabilizing Sirt1 (35). MNA also identified regulated immune responses in the liver by induce IL-10-producing macrophages (36). In this study, the relative abundance of MNA was increased and negatively correlated with serum TBIL and DBIL levels significantly. Although the association analysis also showed positive correlation between the relative abundance of MNA and serum TG level, it could be a false positive, as no significance was observed in the serum TG level (data not shown). The mechanisms how NNMT activity worked and endogenous MNA production initiated remain murky. Our study provided new evidence of the hepatoprotection of MNA, which may protect liver function from damage.

Second, relative abundance of L-palmitoylcarnitine was increased after intervention. L-palmitoylcarnitine is one of the long-chain acyl fatty acid derivative ester of carnitine (37). The low level of L-palmitoylcarnitine was related to the disorder of fatty acid metabolism and further damaged heart, brain and neural (38). A recent follow-up study reported that serum L-palmitoylcarnitine level was lower in HCC 
group compared to control group (39), suggested that the increasement of L-palmitoylcarnitine play a positive role and it may help regulate the lipid profiles. As for the decrease of the relative abundance of Elaidic carnitine, Elaidic carnitine is involved in fatty acid oxidation and it can indicate hepatocytes damage (40). Compound Stigmastane-3,6-dione was extracted from medicinal plants, which showed good selective binding affinity for $\mathrm{CB} 2$ receptor thus could have an anti-inflammatory effect (41). We observed an increase of the relative abundance of Alpha-tocopherolquinone in serum. Alpha-tocopherol (vitamin E), the body's main lipophilic antioxidant, can prevent lipid peroxidation (42) and its primary functions include the termination of the lipid peroxidation cascade induced by free radicals (43). As the main oxidation product of a-tocopherol, Alphatocopherolquinone can be reduced to alpha-tocopherol hydroquinone subsequently (44), which has been reported to play an important antioxidant role (45). Besides, above effects of anti-inflammation, anti-oxidation and fatty acid metabolism may be regulated during the transcription stage, and it then revealed in the metabolites in serum by regulating nuclear factor- $\mathrm{\kappa B}$, nuclear factor erythroid2-related factor 2 and peroxisome proliferators-activated receptor $\alpha$ (46), which provide a new sight in our future study. Above all metabolites identified further provided evidence that COPs' function in hepatoprotection and they may associate with nicotinate and nicotinamide metabolism, fatty acid metabolism, lipid peroxidation and anti-inflammatory action.

Other metabolites such as Levetiracetam, are often used as an antiepileptic drug in clinic, who has few negative side effects impacting the liver (47). In addition, some identified metabolites were first reported as important biomarkers to be associated with liver cancer in the present study, and the relationship between these metabolites and liver cancer remains to be explored in the future.

The present study is the first study to investigate possible effects of COPs on early post-surgery primary liver cancer. However, the current study does have some limitations, we did not verify the specific hepatoprotection mechanism of COPs and it is a single-center study, the number of objects was small. We only conducted a 7-day intervention, which influences the impact of COPs and it may be the reason why most serum indexes did not show significant differences. In the future, we can follow up patients to survey the longterm effects of intervention especially for the improvement of symptoms and quality of life after surgery, and further explore the mechanisms of COPs in protecting liver and other possible health efficacy in vivo and vitro. In addition, the patients included in the present study were with normal $\mathrm{BMI}$, therefore it is still unclear whether it will be the same effects of corn oligopeptides in patients with obese or obesity.

We conclude that the patients had varying degree of liver damage after operation, but the 7-day supplement of COPs has mitigate the injury of liver function, which is consistent with the change of traditional liver injury markers ALT, AST, TBIL, DBIL and liver synthetic function markers PTA, Pre-Alb. Sixteen metabolites were identified and among them, 1-methylnicotinamide, L-palmitoylcarnitine, Elaidic carnitine, Alpha-tocopherolquinone and Stigmastane-3,6-dione indicate that COPs helped improve liver function by affecting fatty acid metabolism, nicotinate and nicotinamide metabolism, lipid peroxidation and antiinflammatory action.

\section{Acknowledgments}

Funding: We sincerely thank all support from National Key Research and Development Program of China (No. 2016YFD0400604).

\section{Footnote}

Reporting Checklist: The authors have completed the CONSORT reporting checklist. Available at https://hbsn. amegroups.com/article/view/10.21037/hbsn-21-116/rc

Data Sharing Statement: Available at https://hbsn. amegroups.com/article/view/10.21037/hbsn-21-116/dss

Conflicts of Interest: All authors have completed the ICMJE uniform disclosure form (available at https://hbsn.amegroups. com/article/view/10.21037/hbsn-21-116/coif). The authors report support from National Key Research and Development Program of China (No. 2016YFD0400604) and have no other conflicts of interest to declare.

Ethical Statement: The authors are accountable for all aspects of the work in ensuring that questions related to the accuracy or integrity of any part of the work are appropriately investigated and resolved. The trial was conducted in accordance with the Declaration of Helsinki (as revised in 2013). The study was approved by the Ethics Committee of National Cancer Center/Cancer Hospital, 
Chinese Academy of Medical Sciences and Peking Union Medical College (permission code: 17-035/1290) and informed consent was taken from all individual participants.

Open Access Statement: This is an Open Access article distributed in accordance with the Creative Commons Attribution-NonCommercial-NoDerivs 4.0 International License (CC BY-NC-ND 4.0), which permits the noncommercial replication and distribution of the article with the strict proviso that no changes or edits are made and the original work is properly cited (including links to both the formal publication through the relevant DOI and the license). See: https://creativecommons.org/licenses/by-nc-nd/4.0/.

\section{References}

1. Bray F, Ferlay J, Soerjomataram I, et al. Global cancer statistics 2018: GLOBOCAN estimates of incidence and mortality worldwide for 36 cancers in 185 countries. CA Cancer J Clin 2018;68:394-424.

2. Global Burden of Disease Cancer Collaboration; Fitzmaurice C, Abate D, et al. Global, Regional, and National Cancer Incidence, Mortality, Years of Life Lost, Years Lived With Disability, and Disability-Adjusted LifeYears for 29 Cancer Groups, 1990 to 2017: A Systematic Analysis for the Global Burden of Disease Study. JAMA Oncol 2019;5:1749-68.

3. Zhou J, Sun HC, Wang Z, et al. Guidelines for Diagnosis and Treatment of Primary Liver Cancer in China (2017 Edition). Liver Cancer 2018;7:235-60.

4. Global Burden of Disease Liver Cancer Collaboration; Akinyemiju T, Abera S, et al. The Burden of Primary Liver Cancer and Underlying Etiologies From 1990 to 2015 at the Global, Regional, and National Level: Results From the Global Burden of Disease Study 2015. JAMA Oncol 2017;3:1683-91.

5. Oh SE, Choi MG, Seo JM, et al. Prognostic significance of perioperative nutritional parameters in patients with gastric cancer. Clin Nutr 2019;38:870-6.

6. Rinninella E, Fagotti A, Cintoni M, et al. Nutritional Interventions to Improve Clinical Outcomes in Ovarian Cancer: A Systematic Review of Randomized Controlled Trials. Nutrients 2019;11:1404.

7. Yan X, Liu L, Zhang Y, et al. Perioperative Enteral Nutrition Improves Postoperative Recovery for Patients with Primary Liver Cancer: A Randomized Controlled Clinical Trial. Nutr Cancer 2021;73:1924-32.
8. Zhou Y, Li Y, Zhou T, et al. Dietary Natural Products for Prevention and Treatment of Liver Cancer. Nutrients 2016;8:156.

9. Ren $\mathrm{Y}$, Yang $\mathrm{Y}, \mathrm{Wu} \mathrm{W}$, et al. Identification and characterization of novel anticoagulant peptide with thrombolytic effect and nutrient oligopeptides with high branched chain amino acid from Whitmania pigra protein. Amino Acids 2016;48:2657-70.

10. Suarez-Jimenez GM, Burgos-Hernandez A, EzquerraBrauer JM. Bioactive peptides and depsipeptides with anticancer potential: sources from marine animals. Mar Drugs 2012;10:963-86.

11. Ganguly A, Sharma K, Majumder K. Food-derived bioactive peptides and their role in ameliorating hypertension and associated cardiovascular diseases. Adv Food Nutr Res 2019;89:165-207.

12. Fernández-Tomé S, Marin AC, Ortega Moreno L, et al. Immunomodulatory Effect of Gut Microbiota-Derived Bioactive Peptides on Human Immune System from Healthy Controls and Patients with Inflammatory Bowel Disease. Nutrients 2019;11:2605.

13. Zhang F, Zhang J, Li Y. Corn oligopeptides protect against early alcoholic liver injury in rats. Food Chem Toxicol 2012;50:2149-54.

14. Lin F, Chen L, Liang R, et al. Pilot-scale production of low molecular weight peptides from corn wet milling byproducts and the antihypertensive effects in vivo and in vitro. Food Chemistry 2011;124:801-7.

15. Zhou C, Hu J, Ma H, et al. Antioxidant peptides from corn gluten meal: Orthogonal design evaluation. Food Chem 2015;187:270-8.

16. Li HM, Hu XIN, Guo P, et al. Antioxidant properties and possible mode of action of corn protein peptides and zein peptides. J Food Biochem 2010;34:44-60.

17. Yamaguchi $M$, Nishikiori F, Ito $M$, et al. The effects of corn peptide ingestion on facilitating alcohol metabolism in healthy men. Biosci Biotechnol Biochem 1997;61:1474-81.

18. Yu Y, Wang L, Wang Y, et al. Hepatoprotective Effect of Albumin Peptides from Corn Germ Meal on Chronic Alcohol-Induced Liver Injury in Mice. J Food Sci 2017;82:2997-3004.

19. She $\mathrm{X}$, Wang F, Ma J, et al. In vitro antioxidant and protective effects of corn peptides on ethanolinduced damage in HepG2 cells. Food and Agricultural Immunology 2016;27:99-110.

20. Jones DP, Park Y, Ziegler TR. Nutritional metabolomics: 
progress in addressing complexity in diet and health. Annu Rev Nutr 2012;32:183-202.

21. Gibbons H, O'Gorman A, Brennan L. Metabolomics as a tool in nutritional research. Curr Opin Lipidol 2015;26:30-4.

22. Gibney MJ, Walsh M, Brennan L, et al. Metabolomics in human nutrition: opportunities and challenges. Am J Clin Nutr 2005;82:497-503.

23. Moazzami AA, Bondia-Pons I, Hanhineva K, et al. Metabolomics reveals the metabolic shifts following an intervention with rye bread in postmenopausal women--a randomized control trial. Nutr J 2012;11:88.

24. Zheng JS, Lin M, Imamura F, et al. Serum metabolomics profiles in response to $\mathrm{n}-3$ fatty acids in Chinese patients with type 2 diabetes: a double-blind randomised controlled trial. Sci Rep 2016;6:29522.

25. Cong WM, Bu H, Chen J, et al. Practice guidelines for the pathological diagnosis of primary liver cancer: 2015 update. World J Gastroenterol 2016;22:9279-87.

26. Mohammadi E, Tamaddoni A, Qujeq D, et al. An investigation of the effects of curcumin on iron overload, hepcidin level, and liver function in $\beta$-thalassemia major patients: A double-blind randomized controlled clinical trial. Phytother Res 2018;32:1828-35.

27. Panahi Y, Kianpour P, Mohtashami R, et al. Efficacy of artichoke leaf extract in non-alcoholic fatty liver disease: A pilot double-blind randomized controlled trial. Phytother Res 2018;32:1382-7.

28. Xiong X, Ren Y, Cui Y, et al. Obeticholic acid protects mice against lipopolysaccharide-induced liver injury and inflammation. Biomed Pharmacother 2017;96:1292-8.

29. Liu X, Cao L, Zhang T, et al. Effect of Remote Ischemic Preconditioning in Patients Undergoing Hepatectomy With Portal Triad Clamping: A Randomized Controlled Trial. Anesth Analg 2019;129:1742-8.

30. Zheng ZJ, Fu J, Zhi F, et al. The effects of interventional therapy on serum HTATIP2/TIP30, B7-H4 and shortterm curative effect in primary hepatocellular carcinoma. Eur Rev Med Pharmacol Sci 2018;22:6778-83.

31. Gong Y, Liu Z, Liao Y, et al. Effectiveness of $\omega-3$ Polyunsaturated Fatty Acids Based Lipid Emulsions for Treatment of Patients after Hepatectomy: A Prospective Clinical Trial. Nutrients 2016;8:357.

32. Shi M, Zhang Z, Xu R, et al. Human mesenchymal stem cell transfusion is safe and improves liver function in acuteon-chronic liver failure patients. Stem Cells Transl Med 2012;1:725-31.

33. Aksoy S, Szumlanski CL, Weinshilboum RM. Human liver nicotinamide $\mathrm{N}$-methyltransferase. cDNA cloning, expression, and biochemical characterization. J Biol Chem 1994;269:14835-40.

34. Sternak M, Khomich TI, Jakubowski A, et al. Nicotinamide N-methyltransferase (NNMT) and 1-methylnicotinamide (MNA) in experimental hepatitis induced by concanavalin A in the mouse. Pharmacol Rep 2010;62:483-93.

35. Hong S, Moreno-Navarrete JM, Wei X, et al. Nicotinamide $\mathrm{N}$-methyltransferase regulates hepatic nutrient metabolism through Sirt1 protein stabilization. Nat Med 2015;21:887-94.

36. Taniki N, Nakamoto N, Chu PS, et al. Intestinal barrier regulates immune responses in the liver via IL-10producing macrophages. JCI Insight 2018.

37. Watanabe H, Kobayashi A, Hayashi H, et al. Effects of long-chain acyl carnitine on membrane fluidity of human erythrocytes. Biochim Biophys Acta 1989;980:315-8.

38. Wang C, Feng R, Li Y, et al. The metabolomic profiling of serum in rats exposed to arsenic using UPLC/Q-TOF MS. Toxicol Lett 2014;229:474-81.

39. Jee SH, Kim M, Kim M, et al. Metabolomics Profiles of Hepatocellular Carcinoma in a Korean Prospective Cohort: The Korean Cancer Prevention Study-II. Cancer Prev Res (Phila) 2018;11:303-12.

40. Liu X, Liu Y, Cheng M, et al. Metabolomic Responses of Human Hepatocytes to Emodin, Aristolochic Acid, and Triptolide: Chemicals Purified from Traditional Chinese Medicines. J Biochem Mol Toxicol 2015;29:533-43.

41. Khedr AI, Ibrahim SR, Mohamed GA, et al. New ursane triterpenoids from Ficus pandurata and their binding affinity for human cannabinoid and opioid receptors. Arch Pharm Res 2016;39:897-911.

42. Burton GW, Foster DO, Perly B, et al. Biological antioxidants. Philos Trans R Soc Lond B Biol Sci 1985;311:565-78.

43. Horton AA, Fairhurst S. Lipid peroxidation and mechanisms of toxicity. Crit Rev Toxicol 1987;18:27-79.

44. Hayashi T, Kanetoshi A, Nakamura M, et al. Reduction of alpha-tocopherolquinone to alphatocopherolhydroquinone in rat hepatocytes. Biochem Pharmacol 1992;44:489-93.

45. Bindoli A, Valente M, Cavallini L. Inhibition of lipid peroxidation by alpha-tocopherolquinone and alphatocopherolhydroquinone. Biochem Int 1985;10:753-61.

46. Valenzuela R, Videla LA. Impact of the Co- 
Administration of N-3 Fatty Acids and Olive Oil

Components in Preclinical Nonalcoholic Fatty Liver

Disease Models: A Mechanistic View. Nutrients

2020;12:499.

Cite this article as: Rong $\mathrm{W}$, Xia H, Zhang $\mathrm{K}$, Zhang $\mathrm{Y}$, Tao C, Wu F, Wang L, Zhang H, Sun G, Wu J. Serum metabolic effects of corn oligopeptides with 7-day supplementation on early post-surgery primary liver cancer patients: a doubleblind randomized controlled trial. HepatoBiliary Surg Nutr 2022;11(6):834-847. doi: 10.21037/hbsn-21-116
47. Khoury T, Chen S, Abu Rmeileh A, et al. Acute liver injury induced by levetiracetam and temozolomide co-treatment. Dig Liver Dis 2017;49:297-300. 